\title{
THE NUMERICAL STUDY OF CHANNEL FLOW IN TURBULENT FREE CONVECTION WITH RADIATION AND BLOWING
}

\author{
Siamak Hosseinzadeh ${ }^{1 *}$, Ali Yari ${ }^{2}$, Morteza Radmanesh $^{3}$ \\ ${ }^{1 *}$ Department of Mechanical Engineering, Islamic Azad University, West Tehran \\ Branch, Tehran, Iran \\ Hoseinzadeh.siamak@gmail.com \\ ${ }^{2}$ Department of Mechanical Engineering, Shiraz University, Shiraz, Iran \\ Ali.Yari.Engineer@gmaail.com \\ ${ }^{3}$ Department of Mechanical Engineering, University College of Rouzbahan, Sari, Iran \\ morteza.radmanesh20@yahoo.com
}

\begin{abstract}
In this article, fluid flow within free convection in a channel, with radiation and blowing from one wall of channel were studied numerically, utilizing Finite volume Method. Turbulent flow with "Low Reynolds Spalart-Allmaras Turbulence Model" and radiation with "Discrete Transfer Radiation Method" had been modeled. In order to have a complete survey, this article has a wide range of study in different domains including velocity and temperature profiles at different locations, turbulent viscosity, shear stress, suctioned mass flow rate in different magnitude of the input Rayleigh, non-dimensional numbers, Reynolds blowing, radiation parameter, Prandtl number, the ratio of length to width and also ratio of opening thickness to width of the channel. In addition, effects of variation in any of the above nondimensional numbers on parameters of the flow are clearly illustrated. At the end resultants had been compared with experimental data which demonstrated that in the present study, results have a great accuracy, relative errors are very small and the curve portraits are in a great agreement with real experiments.
\end{abstract}

\section{KEYWORDS}

Turbulent free convection, Shear stress, Radiation, Blowing

\section{INTRODUCTION}

Investigation on boundary layer of free turbulent convection along a vertical plate is really vital not only in terms of heat transfer mechanism, but also in clarifying the turbulent movement phenomenon. Tsuji et al $[1,2]$ measured the average and turbulent values of velocity and temperature profiles, as well as heat transfer rate and wall shear stress for boundary layer of turbulent free convection flow of the air. His results revealed that the boundary layer of free convection flow has a special turbulent feature that has been fairly seen in other boundary layer of other turbulent flows. On the other hand, the effect of fluid blowing from the surface can be an interesting issue that has been studied for a long time [3]. In the boundary layer, blowing causes an increase in thickness of the boundary level and makes the flow turbulent. The effect of blowing and suction on the boundary layers in free convection flow had been proposed by Eichhorn [4] in which the equations for a vertical wall with site function's temperature were solved using a similarity method. The results showed that blowing and suction in laminar flow has little effect on coefficient of friction. Blowing through a flat plate or a cylinder has been studied both experimentally [5, 6] and numerically [7-12] for many years but also more recently. Similar articles have investigated the issue with other methods (more general modes) 
to evaluate the effect of blowing and suction (about heat transfer inside the channel) on the parameters of the wall shear stresses [13-15]. In many modern systems, such as compact heat exchangers and electronic parts, the mutual effects of turbulent free convection and radiation are considered on each other. Cheng and Muller [15], in a numerical and experimental investigation, examined turbulent free convection flow of air along with thermal radiation within a three-dimensional rectangular channel with a constant hot wall temperature, this study considered a high Reynolds number. They studied the impact of temperature and emission coefficients changes on the velocity and temperature profiles, intake air mass flow rate from end of the channel and heat transfer. Dong and Lu [16] applied LES to a thermally stratified turbulent channel and studied the effects of temperature oscillations on the lower wall. Wang and Pletcher [17] investigated the influence of fluid properties variations in a turbulent channel flow with significant heat transfer. Murata and Mochizuki [18, 19] studied turbulent heat transfer in a two-pass square channel. Lee et al. [20] carried out simulations in a vertical channel with several temperature differences to study the coupling of fluid properties variations and gravity. Chatelain et al. [21] investigated numerical schemes for LES of heat transfer. Recently, problems including heat transfer, radiation, and blowing has been more considered. Abd ElAziz's [22] study represents the effects of thermal-diffusion on the free convection of heat and mass transfer over a permeable plate with suction-blowing, radiation and magnetic field.

The most important point about present study is the fact that this paper considers most of fluid flow parameters including velocity, temperature, wall shear stresses, turbulent viscosity and suctioned mass flow rate from inputs while flow occurs in vertical channels with three parameters free convection, the radiation and blowing in turbulent flow.

\section{Physical Model}

This model consists of a channel with two parallel plates, where height is called $\mathrm{H}$ and width is called L. The left wall is Isolated and the right wall is grooved with constant temperature. Blowing hot gas is done with the same as wall's temperature and uniform velocity from grooved wall, with increase in width and distance. Fluid flow in the channel is created due to the buoyancy force. In the entrance of the channel, fluid is entered with ambient temperature and the atmospheric pressure and hot exhaust gas is discharged to the atmosphere. It is assumed that all surfaces are gray, radiation properties are independent of the wavelength, the radiation coefficient is equal with the absorption coefficient and the fluid is non-participants in the process of radiation.

\section{Physical Model}

All the fluid properties are assumed to be constant, except density terms of free convection in the momentum equations in which had been modeled assuming the Boussinesq. It is assumed that all surfaces are gray, radiation properties are independent of the wavelength, the radiation coefficient is equal with the absorption coefficient and the fluid is non-participants in the process of radiation. The governing equations for steady two-dimensional state of noncompressible flow, using the conservation laws of mass, momentum and energy can thus be written as:

$$
\begin{aligned}
& \left(\rho V_{i}\right)_{, i}=0 \\
& \rho V_{j} \frac{\partial V_{i}}{\partial X_{j}}=\mu \frac{\partial}{\partial X_{j}}\left(\frac{\partial V_{i}}{\partial X_{j}}\right)+g_{i} \beta\left(T-T_{\infty}\right)
\end{aligned}
$$


$\rho C_{p} V_{j} \frac{\partial T}{\partial X_{j}}=-\beta T V_{j} \frac{\partial P}{\partial X_{j}}+\mu\left(\frac{\partial V_{i}}{\partial X_{j}}+\frac{\partial V_{j}}{\partial X_{i}}\right) \frac{\partial V_{i}}{\partial X_{j}}+k_{t h} \frac{\partial}{\partial X_{i}}\left(\frac{\partial T}{\partial X_{i}}\right)$

where all parameters have their conventional definitions. The right wall has Constant temperature and the left wall is isolated, thus radiation flux and flux transport in this boundary are equal $\left(q_{c}=q_{r}\right.$ ). By using Discrete Transfer Radiation Method and "ray tracing" technique, a system of radiation equations are derived, and after solving conservation equations, the flow field and temperature can be determined.

\section{Discrete Transfer Radiation Method AND Ray tracking TECHNIQUE}

The main assumption in this model is the fact that amount of radiation which each element leaves in a certain range of space angles can be estimated by a single beam. In every radiation area, the radiation which is released in discrete values for polar angle $(\theta)$ and horizontal $(\Phi)$ and each beam can be followed to determine the element which is reached and absorbed.

\section{THE STUDY OF NETWORK AND VALIDITY OF RESULTS}

The numerical results for free convective heat transfer in a vertical channel with asymmetric heating and non-blowing wall had been compared with Cheng and Muller's [2] experimental data. In this survey, the channel width ratio $(\mathrm{H} / \mathrm{L})$ is 16 , and the ratio of the whole diameter and gap width with the channel width $(\mathrm{d} / \mathrm{L})$ is 0.008 , respectively radiation ratio is 0.9 and the Rayleigh number equals to $6.5 \times 108$. The act of gridding computational range was according to non-uniform gridding of Form H in Fig. [1]. Due to ensure the independence of numerical results relative to the grid, the three meshes with 80,000,120,000, and 200,000 cells were used to compute. The results of velocity and temperature profiles were similar in all three output channels with high accuracy Fig. [2], and show on average, $12 \%$ and $18 \%$, respectively, different with the experimental data. This is an acceptable accuracy for a numerical solution and confirms the usage of Spalart Allmaras for turbulent flow model. In order to existent of y+ constraint, it is not possible to utilize grids with less than 80000 computational cells. For all presented results, the mesh density or a better one was utilized.

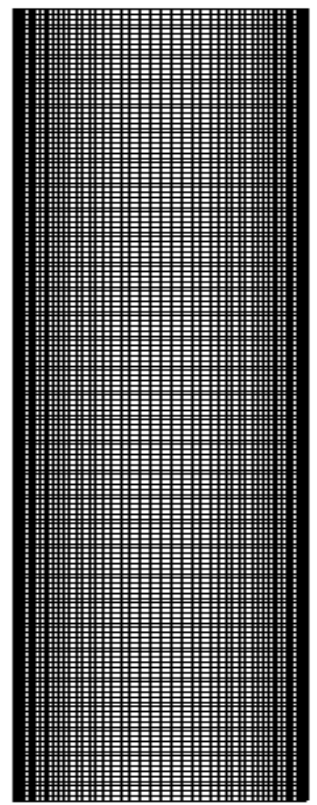


Figure 1. Non-uniform grid for computing zone

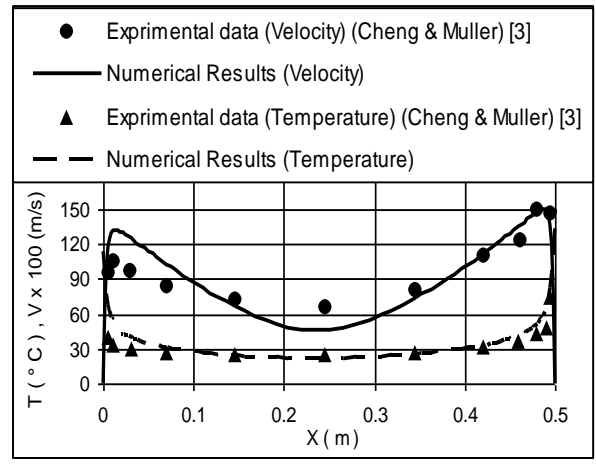

Figure 2. Velocity and temperature profiles in the outlet channel without blowing

$$
\mathrm{H} / \mathrm{L}=16, \mathrm{~d} / \mathrm{L}=0.0008, \varepsilon=0.9, \mathrm{RaH}=6.5 * 10^{\wedge} 8
$$

\section{NUMERICAL RESULTS AND DISCUSSION}

Flow in geometry are considered for the function of six independent dimensionless parameters which include the Rayleigh number for vertical plate's height, RaH, blowing Reynolds, ReB, radiation parameters (number of Stephen ), Stef, Prandtl number, Pr, the ratio of height to width of the channel, $H / L$, and the ratio of tracks thickness to channel width, $d / L$. This article presents resultants of the impacts in three parameters: Rayleigh number for vertical hot plate, blowing Reynolds number and radiation parameter (Stephen number). These three variable parameters are respectively defined as:

$$
R a_{H}=\frac{g \beta \Delta T H^{3}}{\alpha v} \quad \operatorname{Re}_{B}=\frac{\rho V_{B} d}{\mu} \quad, \quad S t e f=\frac{4 \sigma \cdot \varepsilon \cdot T_{1}^{3} \cdot L}{k}
$$

It is important to know that alteration in any of these variables will affect the flow parameters. Thus the problem for different values of the dimensionless numbers has been solved. Quantities of fluid properties and boundary conditions in all cases were considered in such a way that by shifting each parameter, other dimensionless numbers remain constant.

\subsection{Rayleigh Number}

Considering the aim to be investigation of turbulence, the range of Rayleigh number between 109 and 1012 has been studied. Dimensionless shear stress is defined as follows [5].

$\tau_{\omega}^{*}=\frac{V_{B}}{g \beta \Delta T}\left(\frac{\partial u}{\partial x}\right)_{x=L}$

Fixed values for other dimensionless numbers in this mode are selected in the following form:

$$
\operatorname{Re} e_{B}={ }_{5,} \operatorname{Pr}=_{0.72,} \text { Stef }=_{200,} H / L={ }_{4}, d / L=0.008
$$

Fig. [3] Illustrates the dimensionless shear stress values on a part of vertical hot screen. The general trend of changes in shear stress along the plate is the increase which occurs as a result of increase in the fluid velocity in vertical direction and presented by shear stress on warm plates containing blowing, which is consistent with in other papers [7-5]. Jumping at the beginning of 
shear stress graph is due to the edge effects of flow in input of the channel. Marginal effect of the hot gas blowing from holes in the vertical plate causes the shear stress at the upper edge of the whole increase to some extent which makes alternative state for the overall shear stress diagram. The lowering shear stress in each piece of the wall between the two holes can be caused by a relative decrease in the velocity in this fluid area. This slowdown is due to the fact that fluid blown from the puncture above can be a barrier itself to low fluid flow.

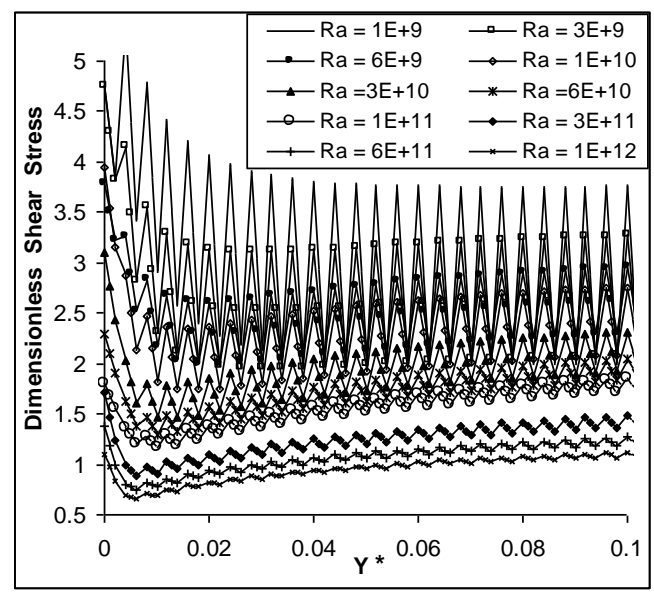

Figure 3. The effect of Rayleigh number on the dimensionless shear stress along the wall containing blowing

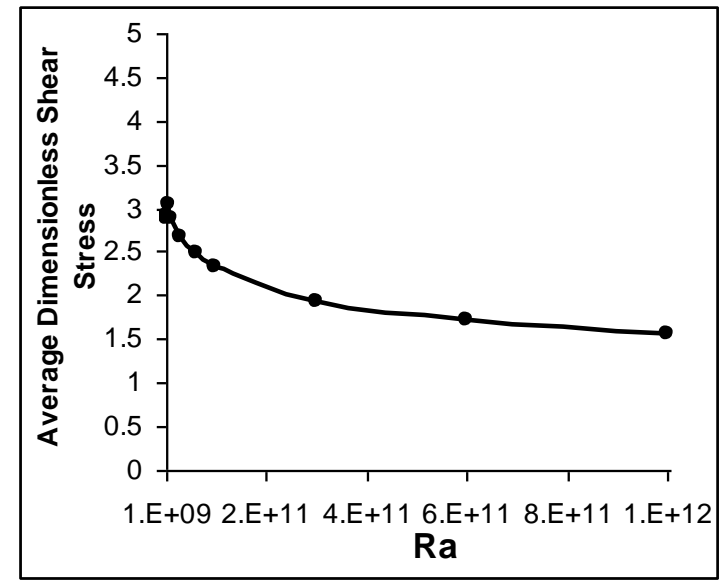

Figure 4. The effect of Rayleigh number on the average dimensionless shear stress along the wall containing blowing

In Fig. [4] The average dimensionless shear stress changes on a heated screen based on Rayleigh number is shown. According the lower Rayleigh values, Rayleigh highly affects shear stress, whereas with increased Rayleigh, the amount of this effect decreases. In high Rayleigh, the effects Rayleigh on the stress, remains constant for highly increased Rayleigh. Fig. [5] Indicates the relative turbulent viscosity variations in viscosity of the fluid with Rayleigh number. It can be observed that in low Rayleigh numbers, the only turbulent flow is at the end the channel in hot wall blowing wall, which reaches the maximum level in the external opening. However, with the increase in the Rayleigh number, turbulent flow occurs earlier and in addition to the hot wall, the boundary layer which was caused by the isolated wall is also turbulent. In Rayleigh number 1012, almost the entire channel is turbulent in which, the 
turbulent viscosity is approximately 300 times more than fluid viscosity. This turbulence has a significant effect on the heat transfer and wall shear stress.
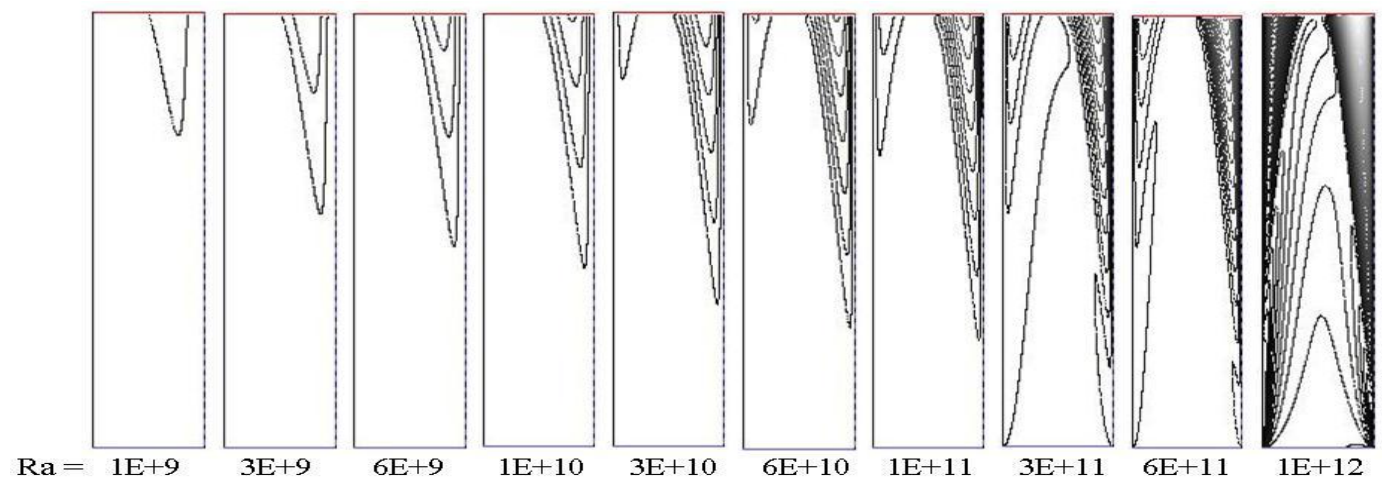

Figure 5. The effect of Rayleigh changes on Turbulent Viscosity level with fluid viscosity in the channel

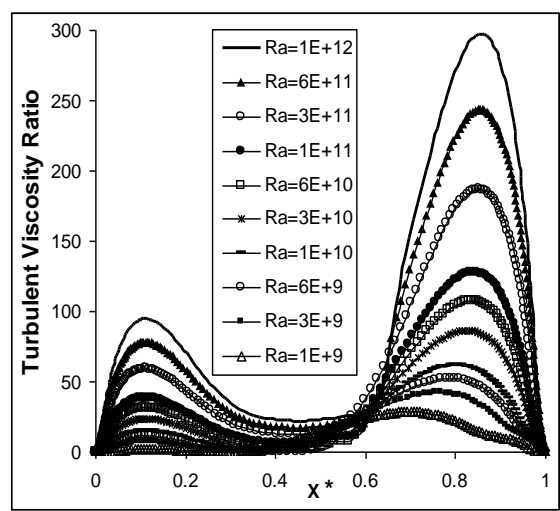

Figure 6. The effect of Rayleigh on Turbulent Viscosity level with fluid viscosity in the outlet

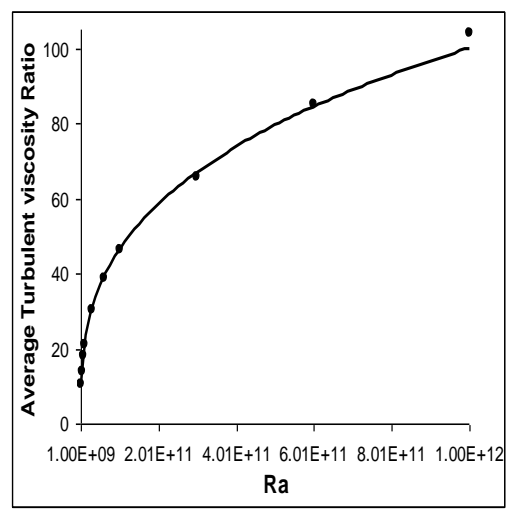

Figure 7. The effect of Rayleigh on the average turbulent viscosity ratio with fluid viscosity in the outlet

Fig. [6]shows the ratio of turbulent viscosity to the viscosity of the fluid output opening which has only one relative maximum in low Rayleigh numbers (which approximately reaches 30 ). However, as the Rayleigh gradually increases, the boundary layer of the insulated wall will become significantly turbulent and the graph has two relative peaks. For Rayleigh numbers which are greater than 1010 , in $(0.6=\mathrm{X} *)$, fracture is evident which is caused by the backflow 
of output opening. Given that flow in a channel is a suction flow caused by free convection, if the discharge from suctioned opening inlet fails to decrease the pressure in the channel, the flow will return. In Fig. [7], the average relative turbulent viscosity against the Rayleigh number variations is drawn, which indicates that the turbulent viscosity dependence on Rayleigh number is an exponential dependence with negative concavity. To define the reference velocity and dimensionless velocity, we applied the definition of reference velocity in free convection on a vertical plate. For such a plate, the reference velocity is defined as $\frac{\alpha}{H} R a^{\frac{1}{2}}$ [23]. For the investigated problem, the output velocity caused by blown discharges, the value will also be added in the channel output. Thus the sequence of reference velocity and dimensionless velocity are defined as:

$$
\begin{aligned}
V_{\text {ref }} & =\left(\frac{\alpha}{H} R a_{H}^{\frac{1}{2}}+\frac{H}{2 L} V_{B}\right) \\
V^{*} & =V \times\left(\frac{\alpha}{H} R a_{H}^{\frac{1}{2}}+\frac{H}{2 L} V_{B}\right)^{-1}
\end{aligned}
$$

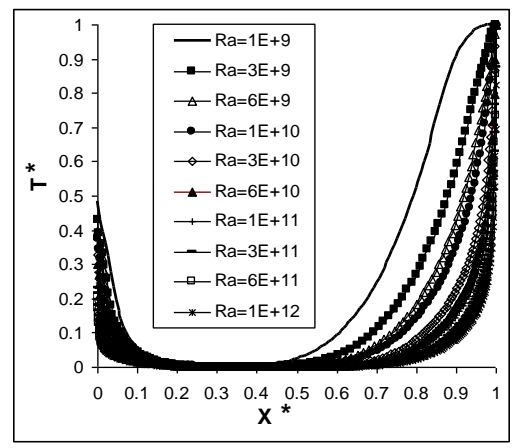

Figure 8. The effect of Rayleigh number on the dimensionless temperature in the outlet channel

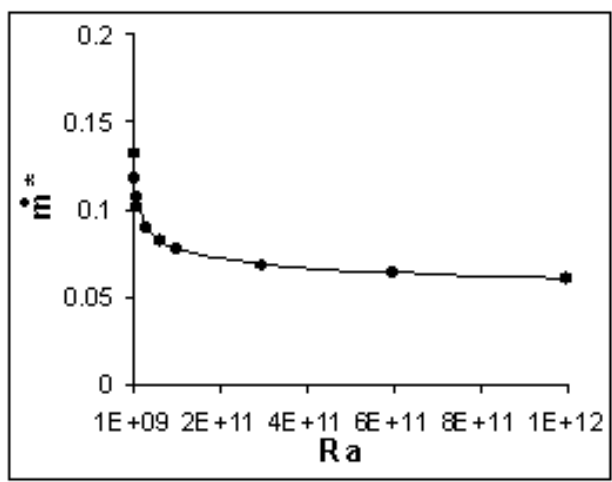

Figure 9. The effect of Rayleigh number on the dimensionless mass flow rate suctioned from the channel input

Fig. [8] Displays dimensionless velocity profile variation as the Rayleigh changes. It can be seen that the overall pattern of the diagram remains constant with the increase in Rayleigh, but the impact of Rayleigh on velocity decreases which is considered in shear stress. Also, with the increase of Rayleigh, the relative thickness of the boundary layer reduces and the flow gets closer to the wall. In order to make the input suctioned mass discharge dimensionless, the first 
statement of the velocity statement is applied. Thus, the suctioned dimensionless mass discharge will be as follows:

$$
\dot{m}^{*}=\dot{m} \times\left(\frac{\rho_{0} L \alpha}{H} R a_{H}^{\frac{1}{2}}\right)^{-1}
$$

In which $\rho_{0}$ is the density of suctioned fluid in the input temperature. Figure (9) shows the relation between the dimensionless mass suctioned from the channel input with Rayleigh number. It can be perceived that the slope of the diagram is very high whereas it decreases with the increase in Rayleigh number. As shown in figure (10) as the Rayleigh number increases, the floating power goes up, the velocity of the fluid flow increase and the relative temperature in the channel decreases.

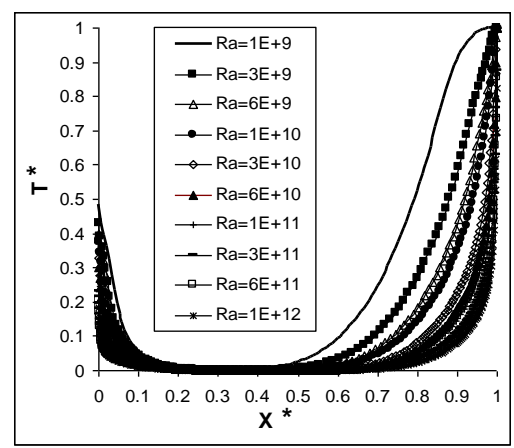

Figure 10. The effect of Rayleigh number on the dimensionless temperature in the outlet channel

\subsection{Blowing Reynolds Number}

In order to investigate the effects of Reynolds number, a range of Reynolds number between 0 and 15 were studied. Fixed values for other dimensionless numbers were selected this way:

$$
R e_{B}={ }_{1010}, P r=0.72, \text { Stef }=_{200}, H / L={ }_{4}, d / L=0.008
$$

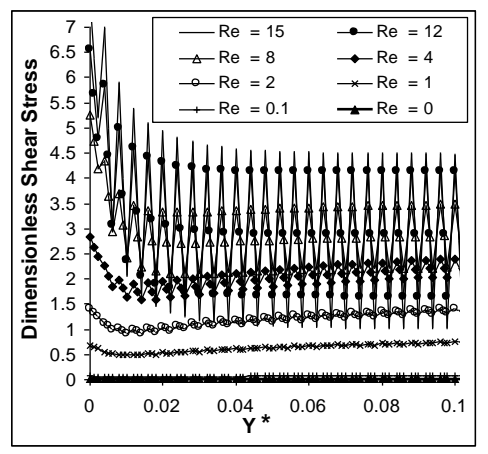

Figure 11. The effect of Reynolds number on the dimensionless shear stress along the wall containing blowing 


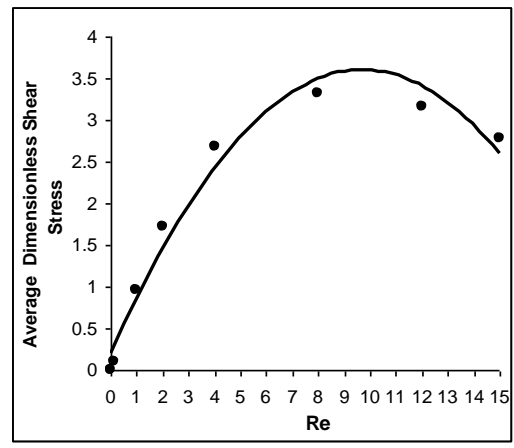

Figure 12. the effect of Reynolds number on the average dimensionless shear stress along the wall containing blowing

By drawing a dimensionless shear stress on hot wall based on Reynolds number, it can be observed in figure (11) that the dimensionless shear stress diagram increased up to almost 8 Reynolds number and has an upward trend. However, this increase cannot be observed for higher Reynolds number. In addition, as the Reynolds number increases, the edge effect increases which causes the range fluctuations in shear stress to rise. Figure (12) shows the average shear stress on the wall with Reynolds number variations which changes in parabolic function with negative concavity. Considering the different states, some interesting results can be achieved. First, we consider that Reynolds has a linear relation with velocity, and the dimensionless shear stress has a direct correlation velocity and the shear stress of the wall. If the increase in Reynolds is merely the results of increase in velocity, then the negative concave shows a decrease in the wall shear stress with the increase of velocity. The result is due to the fact that the blown warm fluid pushes back the flowing fluid in the channel and blocks the creation of shear stress between the fluid layers in the channel and the well. Also, as the velocity and consequently the Reynolds increases, the impact of the pushing back increases too. If the increase in Reynolds is associated with a constant speed, it can be concluded that increase in Reynolds (increase in density or decrease in viscosity diameter) can increases the shear stress. However, this trend continues as long as the boundary layers of the two walls did not meet. In Reynolds number more than 8 , in which the boundary layers meet, the impact of the increase in Reynolds (with constant velocity), becomes reverse.
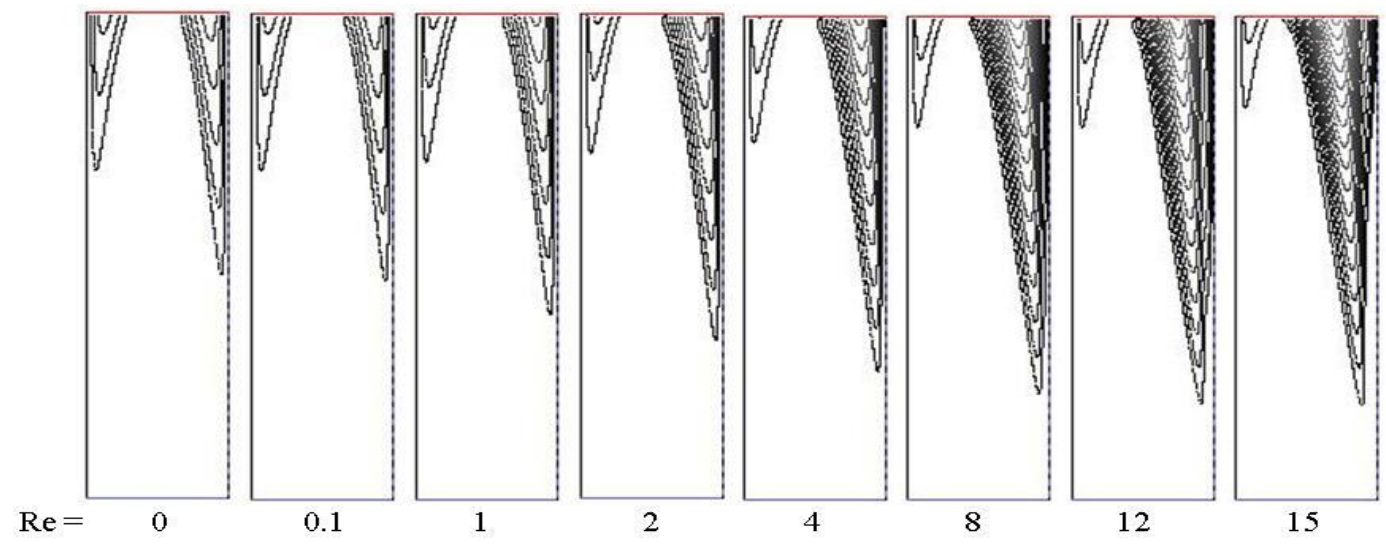

Figure 13 : The effects of Reynolds variation on the turbulent viscosity ratio to fluid viscosity in the channel.

In Fig. (16) The variations in output dimensionless velocity is shown with respect to the variations in Reynolds number. Although the blowing Reynolds variations do not have a 
significant impact on the boundary layer of the insulated wall, it causes the boundary layer of the heated blowing wall to extend strongly. Increase in the blowing Reynolds not only augments the amount of diagram's quantity curve, it will also increase the distance of maximum point of the graph's curve from the right part of wall in which Rayleigh number growths inversely. Fig. (17) Demonstrates that Increase in Reynolds Number can also augment the negative concavity of the dimensionless mass flow of the fluid suction from the inlet opening. At lower Reynolds numbers range, there is higher effect of increasing blowing Reynolds on the suctioned mass flow, but its impact reduces gradually. The reason of mass flow is evident according to what has been already mentioned in shear stress variation. The impact of blowing Reynolds on the output fluid temperature is clearly illustrated in Fig. (18). More blowing of the heated fluid makes increase in the temperature of the channel's right half.

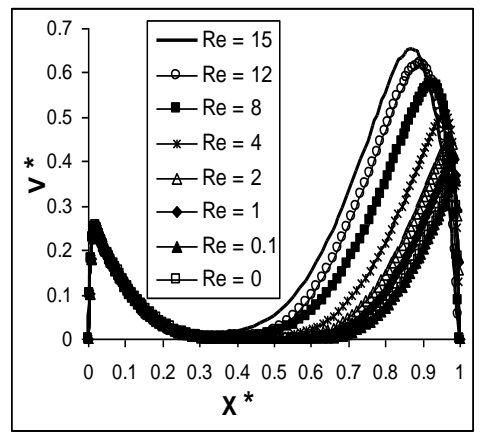

Figure 16. : The effect of Reynolds on the dimensionless velocity in the outlet channel.

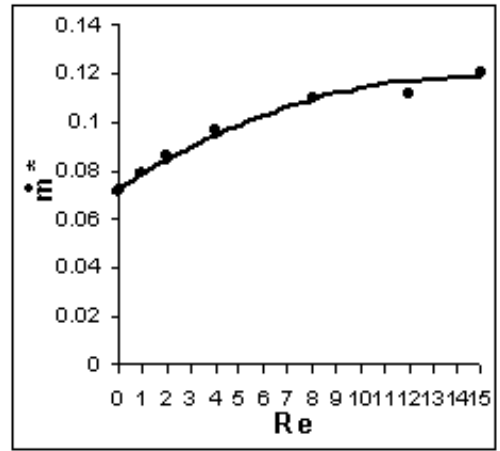

Figure 17. : The effect of Reynolds on the dimensionless mass flow suctioned from the channel input

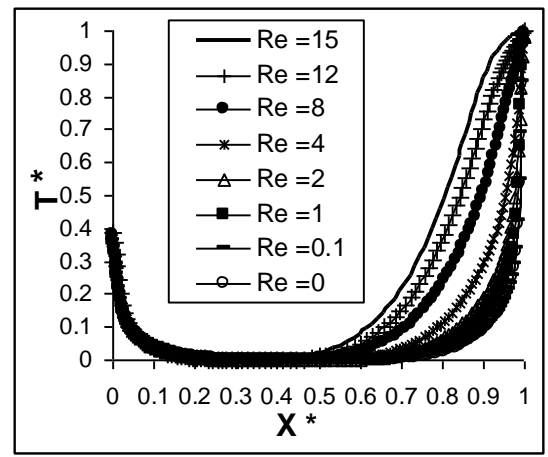

Figure 18. : The effect of Reynolds on the dimensionless temperature in channel output 


\subsection{Stephen Numbers}

In order to study the radiation parameters, a range of Stephen numbers from 0 to 250 has been investigated. The fixed values for other dimensionless numbers in this state were selected by this method:

$$
R e_{B}={ }_{1010,} \operatorname{Re}_{B}={ }_{5,} \operatorname{Pr}={ }_{0.72}, H / L={ }_{4}, d / L=0.008
$$

Figs (19), (20) and (21) show that the radiation parameters (Stephen number) had an insignificant impact on the dimensionless shear stress wall with blowing, while an increase in Stephen number causes a rise in the dimensionless shear stress of the insulated wall with parabolic function.

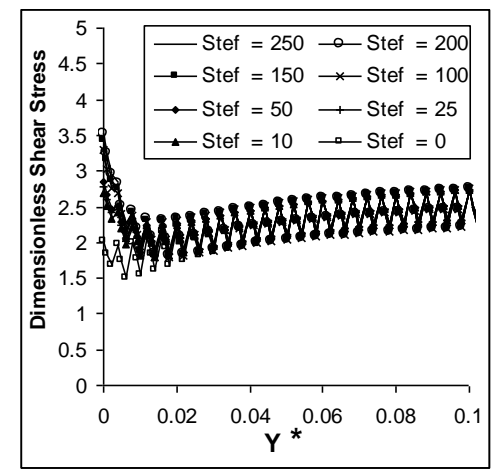

Figure 19: The effect of Stefan number on the dimensionless shear stress along the wall containing blowing

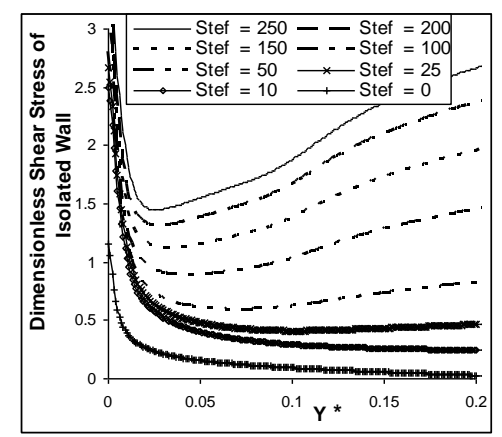

Figure 20: The effect of Stefan number on the dimensionless shear stress along the isolated wall

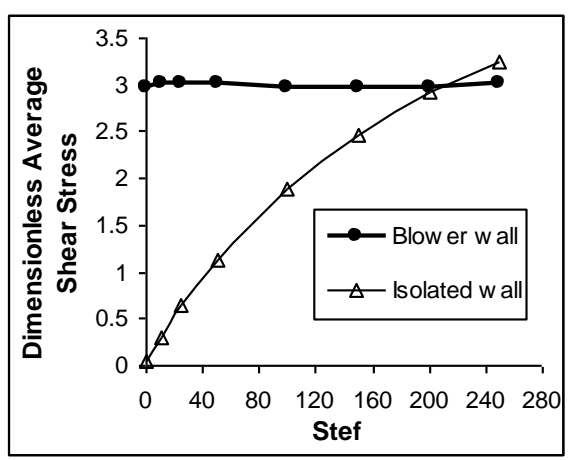


Figure 21: The effect of Stefan number on the average dimensionless shear stress along the wall containing blowing and isolation

Figs. (22) And (23) show the relative turbulent output viscosity in which the pattern and values are constant in the right part of the graph (except for the radiation less state) whereas augmentation of radiation parameters causes' growth in turbulence of the channel's left part.

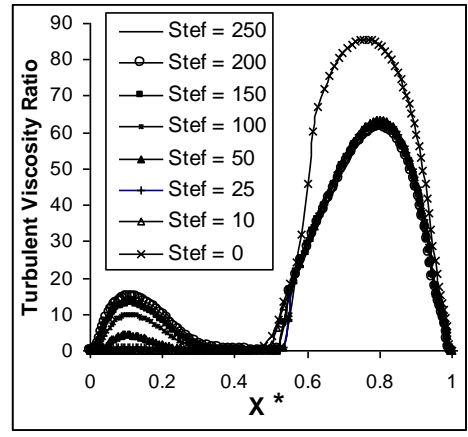

Figure 22: The effect of Stefan number increase on the ratio of turbulent viscosity to the fluid viscosity in the outlet

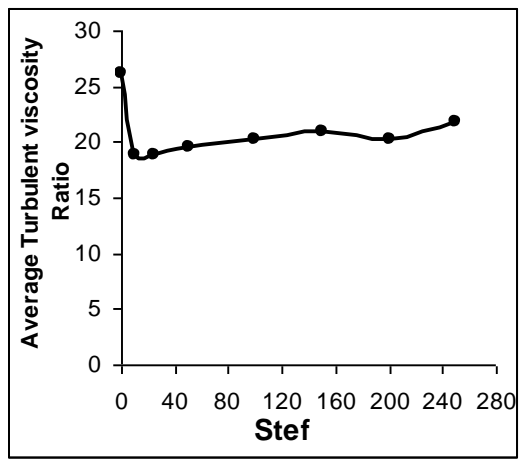

Figure 23 : the effect of Stefan number on the average ratio of turbulent viscosity to the fluid viscosity in the outlet channel.

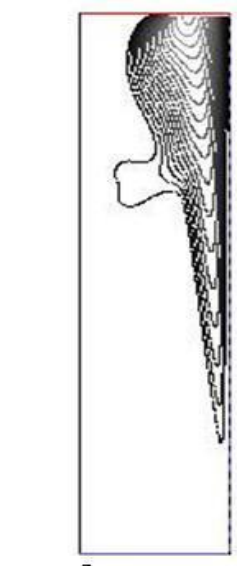

Stef $=0$

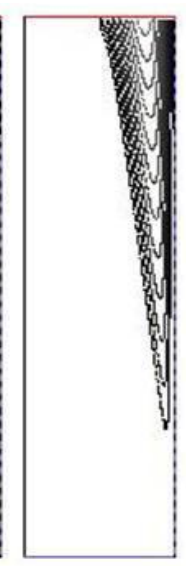

10

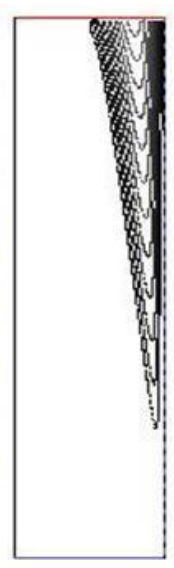

25

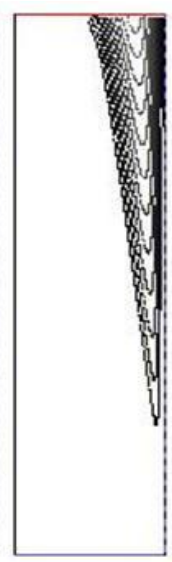

50

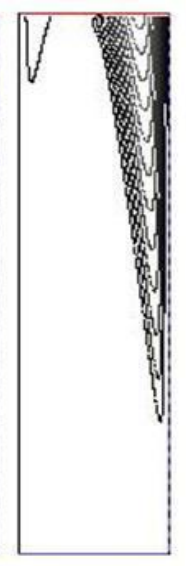

100

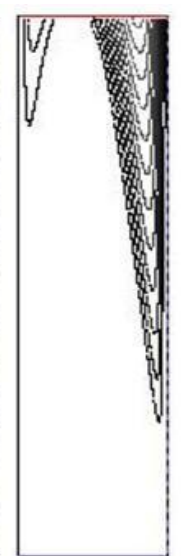

150

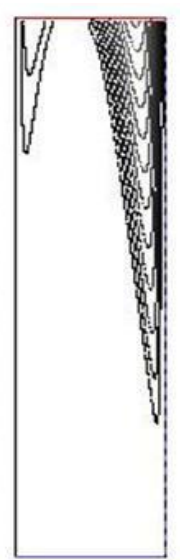

200

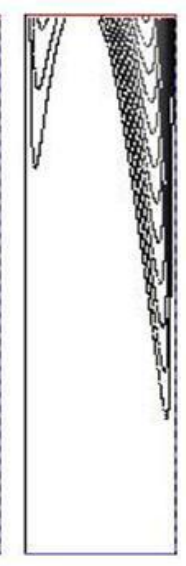

250

Figure 24 : he effect of Stefan number variability on the ratio of turbulent viscosity to the viscosity of the fluid in the channel 
Fig. (24) Also illustrates that, except for the Stephen number in Zero mode, radiation does not have a significant impact on the turbulent flow. In non-radiation mode, there is a return flow in the left part of channel which causes graph pattern to change completely. However, despite the radiation, there is an upward flow in the left part of the graph which blocks the return flow.

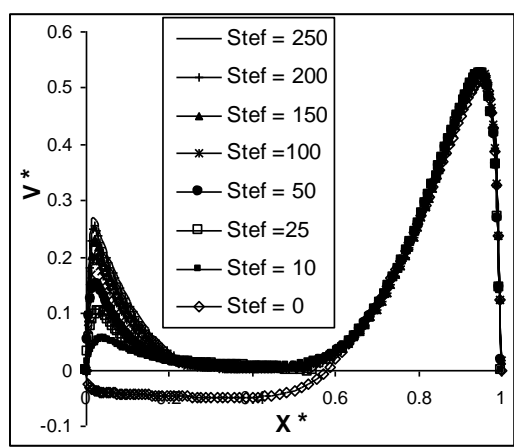

Figure 25: the effect of Stephan number on the dimensionless temperature in the outlet channel.

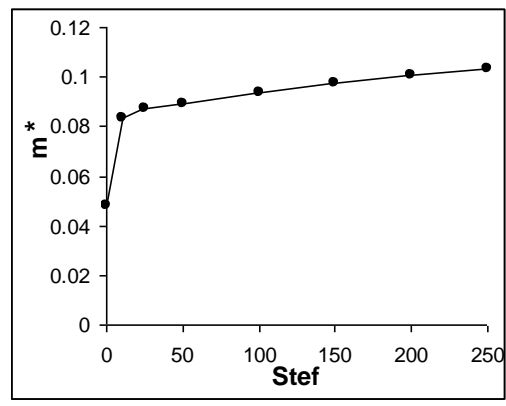

Figure 22: the effect of Stephan number on the dimensionless mass flow suctioned from the input channel

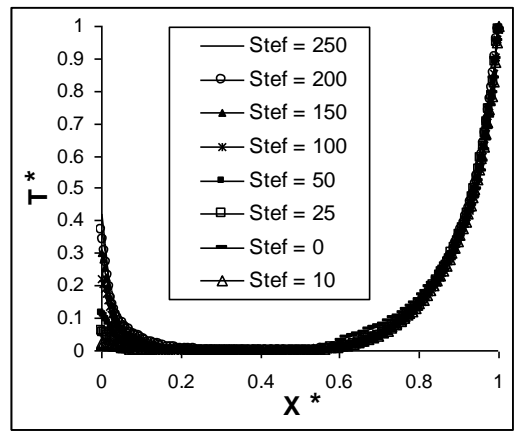

Figure 22: : the effect of Stephan number on the dimensionless temperature in the outlet channel

Fig. (25) Shows the output velocity of dimensionless profile in channel, which is drawn for different Stephen numbers. In general, it can be observed that decrease in Stephen number can causes decline in values of the left part, but does not have any effect on the boundary layer of the right part in channel. The negative values of velocity in non-radiation mode indicate existence of return flow. Fig. (26) Shows that despite the line radiation, the amount of dimensionless mass flow which is suctioned from the input opening changes with the Stephen 
number. The reason for decrease in the graph values in non-radiation mode can be the existence the return flow. Fig. (27) also illustrates the changes in relative output temperature which are caused by the changes in Stephen number in which the dependence of the temperature in boundary layer of the left channel on the radiation parameters is clearly visible.

\section{CONCLUSION}

In this paper, the effect of radiation and blowing on free convection of fluid flow parameters was studied in a vertical channel. The results clearly demonstrate that the radiation process acts independent of blowing and free convection on the radiation wall. Blowing from wall has a double effect on shear stress of the radiation wall which led to cause increase in lower Reynolds numbers, and a decrease in shear stress in higher Reynolds numbers. Also, blowing increases the turbulence and the flow rate suctioned from input of the channel. In addition, effect of the Rayleigh number is higher on flow in lower Rayleigh number and the effect decreases as the Rayleigh number goes up..

\section{REFERENCES}

[1] Tsuji, T., Nagano, Y., "Characteristics of a Turbulent Natural Convection Boundary Layer along a Vertical Flat Plate", Int. J. Heat Mass Transfer, vol.31, pp. 1723-1734, (1988).

[2] Tsuji, T., Nagano, Y., "Turbulence measurements in natural convection boundary layer along a vertical flat plate", Int. J. Heat Mass Transfer, Vol. 31, pp. 2101-2111, (1988).

[3] Gupta, A. S., Misra, J.C., Reza, M., "Effects of suction or blowing on the velocity and temperature distribution in the flow past a porous flat plate of a power-law fluid", J. of Fluid Dynamics Research, Vol. 32, pp. 283-294, (2003).

[4] Eichhorn, R., "The effect of mass transfer on free convection", J. of Heat Transfer, Vol. 82, pp. 260-263, (1960).

[5] Bellettre, J., Bataille, F., Rodet, J. C., Lallemand, A., "Thermal behaviour of porous plates subjected to air blowing", AIAA J. Thermophys. Heat Transfer 14 (4) (2000) 523-532.

[6] Whitten, D.G., Moffat, R.J., Kays, W.M., "Heat transfer to a turbulent boundary layer with nonuniform blowing and surface temperature", in: 4th Int. Heat Transfer Conf., 1970.

[7] Bellettre, J., Bataille, F., Lallemand, A., "A new approach for the study of turbulent boundary layers with blowing", Int. J. Heat Mass Transfer 42 (15) (1999) 2905-2920. [2] Tsuji, T., Nagano, Y., "Turbulence measurements in natural convection boundary layer along a vertical flat plate", Int. J. Heat Mass Transfer, Vol. 31, pp. 2101-2111, (1988).

[8] Brillant, G., Bataille, F., Ducros, F., "Large-eddy simulation of a turbulent boundary layer with blowing", Theor. Comput. Fluid Dyn. 17 (2004) 433-443.

[9] Mathelin, L., Bataille, F., Lallemand, A., "Near wake of a circular cylinder submitted to blowing - I boundary layers evolution", Int. J. Heat Mass Transfer 44 (2001) 3701-3708.

[10] Mathelin, L., Bataille, F., Lallemand, A., "Near wake of a circular cylinder submitted to blowing - II impact on the dynamics", Int. J. Heat Mass Transfer 44 (2001) 3701-3708.

[11] ] N. Shima, Prediction of turbulent boundary layers with a secondmoment closure: Part i effects of periodic pressure gradient, wall transpiration and free-stream turbulence, Trans. ASME J. Fluid Eng. 115 (1) (1993) 56-63.

[12] A.P. Silva-Freire, D.O.A. Cruz, C.C. Pellegrini, Velocity and temperature distributions in compressible turbulent boundary layers with heat and mass transfer, Int. J. Heat Mass Transfer 38 (13) (1995) 2507-2515.

[13] Merkin, J. H., "Free Convection with Blowing and Suction", Int. J. Heat Mass Transfer, Vol. 15, pp. 989-999, (1972). 
[14] Vedhanayagam, M., Altenkirch R. A., Eichhorn, R., "A Transformation of the Boundary Layer Equations for Free Convection Past a Vertical Flat Plate with Arbitrary Blowing and Wall Temperature Variations", Int. J. Heat Mass Transfer, Vol.23, pp. 1286-1288, (1980).

[15] Brouwers, H. J. H., "The Film Model Applied to Free Convection Over a Vertical Plate with Blowing or Suction", Int. J. Heat Mass Transfer, Vol. 35, No. 7, pp. 1841-1844, (1992).

[16] Cheng, X., and Muller, U., "Turbulent natural convection coupled with thermal radiation in large vertical channels with asymmetric heating", Int. J. Heat Mass Transfer, Vol. 41, No. 12, pp. 1681-1692, (1998).

[17] Dong, Y.-H., Lu, X.-Y., "Large eddy simulation of a thermally stratified turbulent channel flow with temperature oscillation on the wall", International Journal of Heat and Mass Transfer 47, 2109-2122, (2004).

[18] Wang, W.P., Pletcher, R.H., 1996. On the large eddy simulation of a turbulent channel flow with significant heat transfer. Physics of Fluid 8 (12), 3354-3366.

[19] Murata, A., Mochizuki, S., 2004a. Large eddy simulation of turbulent heat transfer in a rotating two-pass smooth square channel with sharp 180_turns. International Journal of Heat and Mass Transfer 47, 683-698.

[20] Murata, A., Mochizuki, S., 2004b. Effect of rib orientation and channel rotation on turbulent heat transfer in a two-pass square channel with sharp 180_ turns investigated by using large eddy simulation. International Journal of Heat and Mass Transfer 47, 2599-2618.

[21] Lee, J.S., Xu, W., Pletcher, H., 2004. Large eddy simulation of heated vertical annular pipe flow in fully developed turbulent mixed convection. International Journal of Heat and Mass Transfer 47, 437-446.

[22] Chatelain, A., Ducros, F., Métais, O., 2004. LES of turbulent heat transfer: proper

[23] Abd El-Aziz, M., "Thermal-diffusion and diffusion-thermo effects on combined heat and mass transfer by hydromagnetic three-dimensional free convection over a permeable stretching surface with radiation", Physics Letters A, Vol. 372, pp. 269-276, (2008).

[24] Bejan, A., "Convection Heat Transfer", Third Edition, John Wiley \& Sons, New Jersey, (2004). [2] Tsuji, T., Nagano, Y., "Turbulence measurements in natural convection boundary layer along a vertical flat plate", Int. J. Heat Mass Transfer, Vol. 31, pp. 2101-2111, (1988). 


\section{Authors}

Siamak. Hosseinzadeh was born in August 22nd 1985 in Sari,Mazandaran. He received his Ms. degree in mechanical engineering (Energy Conversion) from South Tehran Branch in 2010. He also received his BSc degree in thermal fluids from Islamic Azad University Sari Branch, Mazandaran in 2007. He did his military service in Manufacturing, Mining and Trade organization as an industry expert from 2010 to 2012. He is currently a university lecturer in Islamic Azad Universities in Mazandaran, Iran. The teaching courses included Mechanical technology, Automotive and Machinery, Power Plant Technology, Installation Technology, Chemical Industry, Architecture, Power Electronics, Project Management and Executive Management. He also was a Technical Office Manager (Head Office) in Nogostaran Construction Installation Company in Tehran from 2007 to 2011.

ali yari was born in Septembr 21nd 1983 in Qaemshahr,Mazandaran. He received his Ms. degree in mechanical engineering (Energy Conversion) from Shiraz University, Shiraz, Iran. He also received his BSc degree in thermal fluids from in thermal fluids from BabolNoshirvani University of Technology, Babol, Mazandaran. He did his military service in Education as a Soldier teacher from 2010 to 2012. He is currently a university lecturer in Universities in Mazandaran, Iran.

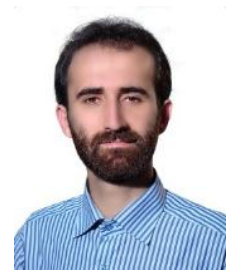

Morteza Radmanesh was born in August 29nd 1995 in Tehran,Tehran. He is a student in Mechanical (mechanical installation) at Institute of Higher Education Roozbahanal in Sari, Mazandaran. He is also the designer of mechanical systems in Company of Industry facility in Sari,Mazandaran, Iran.

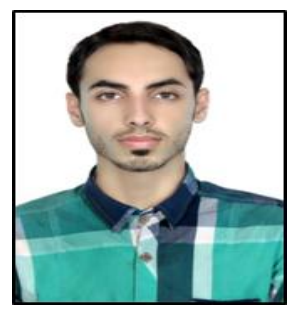

